\title{
Furniture Positioning Using Augmented Reality
}

\author{
Abhijeet R. Raipurkar' ${ }^{1}$ Manoj B. Chandak ${ }^{2}$ and Anshit Raika ${ }^{3}$ \\ ${ }^{1,2}$ Department of Computer Science and Engineering, Shri Ramdeobaba \\ College of Engineering and Management, Nagpur \\ ${ }^{3}$ Amazon India, Chennai
}

\section{ABSTRACT}

Buying furniture has been a daunting task for every family. Checklist associated with buying furniture is a long one and matching of the furniture item in their house is a very important aspect. The customers have a hard task in imagining the matching of the furniture item in their house. Here the concept of Augmented Reality comes to the aid of customers. With the help of augmented reality the customer can actually view how the furniture will be look at their house and based on that make intelligent purchase. This application will be beneficial to the furniture sellers as they will be able to market their products in a better and more efficient way. Thus the sellers will be able to provide better service to customers and grow their business. Intended to build a UNITY3d model based android application that will help customers visualize the furniture item set at their place. A sample booklet for the furniture will be provided to the customers by the seller. This booklet will have all the images of furniture item sets and customer can scan this and view the $3 \mathrm{~d}$ model of the items at their home and can check which item suits them most. The use of Vuforia is very important along with the unity $3 \mathrm{~d}$ as Vuforia stores the marker images which will be used as the marker for projecting the $3 \mathrm{~d}$ model of the furniture item sets.

\section{KEY WORDS: AUGMENTED REALITY, VUFORIA , UNITY 3D, MARKERS.}

\section{INTRODUCTION}

Furniture industry is growing at a fast pace. But the main problem that customers faces is that they find difficult to imagine how the item will match \& fit in their home. Also the sellers have a problem in making the customers understand which furniture item will be best for them. Thus our augmented reality based application solves this problem of customers about the furniture items and positioning of furniture items at there home. The main objective of the project is to develop user friendly mobile

\section{ARTICLE INFORMATION}

Received 12th Oct 2020 Accepted after revision 31st Dec 2020 Print ISSN: 0974-6455 Online ISSN: 2321-4007 CODEN: BBRCBA

Thomson Reuters ISI Web of Science Clarivate Analytics USA and Crossref Indexed Journal

\section{Clarivate $\begin{gathered}\text { Cnalytics } \\ \text { Crossef }\end{gathered}$}

NAAS Journal Score 2020 (4.31) SJIF: 2020 (7.728)

A Society of Science and Nature Publication,

Bhopal India 2020. All rights reserved.

Online Contents Available at: http//www.bbrc.in/

Doi: http://dx.doi.org/10.21786/bbrc/13.14/17 application which can project 3D model of furniture items.

Problem Definition: To design a prototype system that is intended to aid the people by providing an android application to see how the room will look with new furniture without actually buying or moving real furniture while simulating an arrangement of furniture.

Use of Application: This application has a wide area of implementation. Each seller can have his own application customized as per his needs and set of furniture items available with him. There is also scope for advertisement videos for the seller based on the marker. This application can be made available for Android as well as iOS users. It will replace the traditional ways of imagining the fit of the furniture item by the customer. It will help customer make better choices in selection of furniture items and help the sellers expand their business. It will also solve the issue of positioning of furniture at customers home

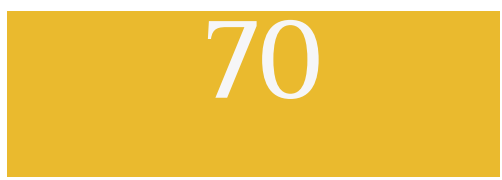


or office in case of large number of furniture items and aid them in quick decision making which will save time as well as cost.

\section{Literature Survey}

Augmented Reality: Augmented reality (AR) can be seen as a technology where the objects in the real world are enhanced by computer-generated mathematical knowledge, sometimes in many emotional senses, including visual, tangible, abstract, visual and virtual reality. A key feature of augmented reality is the approach that integrates parts of the digital world into the real-world individual's perception. It is not only the general presentation of data, but also the consolidation of captivating sensations that appear to be natural components of the surroundings.

\section{Challenges In Augmented Reality}

Hardware Issues: Most of the increased reality headsets are huge and very expensive for the people. Adding to this, most of the available headsets that support AR need to be connected to a computer thus hampering the entire experience and making it confined and bounded. On the other hand, the end users can use their smartphone or tablet for AR related applications. However, one of the problems with smartphones is that the scenes are not displayed properly. Mobile sensors such as accelerometers can be interrupted by electrical interference, which is often seen in urban areas. Majority of the available smartphones or tablets are made to capture the two dimensional images and are not able to give three dimensional images. Making a note of the above mentioned problems, the hardware that is needed for technologies like AR needs some expansion before moving towards mass adoption.

Limited Content: Creating dynamic content and engaging it as per the requirements is one of the challenges for augmented reality. Strainers \&t some recreational games used in social networking sites such as "Snapchat" and "Instagram" are one of the major contents for AR devices. AR can also be used for promoting businesses but it can be extremely complicated and expensive. The developers of the technology like augmented reality should go for some high-functionality use cases so that AR can be a useful technology for end-users on diurnal

\section{METHODOLOGY}

Th methodology includes the procedure for implementation of the project and a brief explanation of why it will be useful for implementing the proposed system. This module will show the usage of Vuforia and Unity3d for linking the $3 \mathrm{~d}$ models to their respective markers.

The system frame diagram displays the flow chart on the entire process that is required for implementing the augmented reality application.

- Vuforia database creation: Images of items that will act as marker for the 3D models of furniture items will be stored. A key is generated which is to be entered in unity3D in order to link it with Vuforia database.

- 3D Model creations: In this step, the 3D model will be created for respective furniture items using Blender tool. The 3D models for the ffbx format will be stored under the projects folder in unity3D.

- Assigning target image to the 3D Models: A target image will be assigned to the 3D models. Application will scan the markers from the image and project the 3D model.

- C\# scripts for Rotation and scaling: The user should be able to move scale and rotate the $3 \mathrm{D}$ model generated as per their requirements. C\# scripts are written so that the user can scale rotate and move the $3 \mathrm{D}$ model by his fingers.

- Building environment and deploying the APK: Adding of the SDK and the JDK path of the system to the Unity 3D software. The Unity 3D software uses these paths in order to build the APK file of the application, since the entire android application is java based and thus it required the specifications of the path of SDK and JDK.

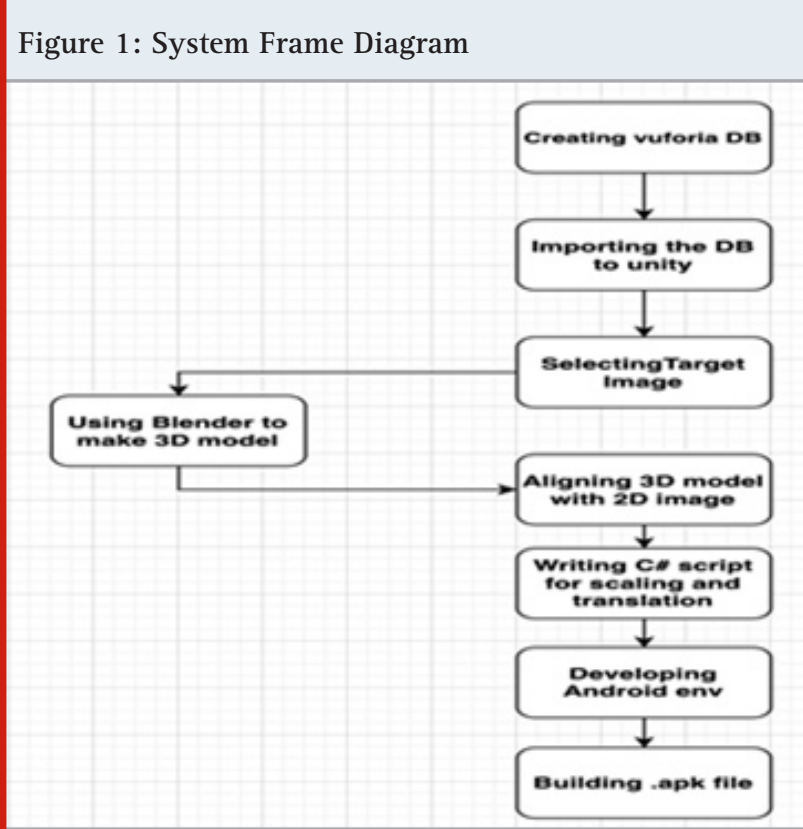

System Implementation Methodology

Unity 3d: Unity is a multi-platform, integrated IDE for scripting games, and working with $3 \mathrm{D}$ virtual worlds including:

- Game Engine

- 3D terrain editor

- 3D object animation manager

- GUI System

Unity gives users the ability to create games and experiences in both $2 \mathrm{D}$ and $3 \mathrm{D}$, and the engine offers a primary scripting API in C\#. Prior to C\# being the primary programming language used for the engine, it previously supported Boo, which was removed with the release of Unity 5 and a version of JavaScript called Unity-Script, which was deprecated in August 2017, after the release of Unity 2017.1, in favour of C\#. Within 2D 
applications, Unity allows importation of sprites and an advanced 2D world renderer. For 3D applications, Unity allows specification of texture compression and resolution settings for each platform that the game engine supports, and provides support for bump mapping, reflection mapping, parallax mapping, screen space ambient occlusion (SSAO), dynamic shadows using shadow maps, render-to-texture and full-screen postprocessing effects.

Vuforia: Since augmented reality is used for developing many applications, thus there is a need of a software development kit for AR. This software development kit used for AR is called Vuforia. Vuforia enables the creation and development of applications by using computer vision technology to trace, track locate and identify three dimensional targets in real time. This allows the developers to potentially place the three dimensional object over the planer two dimensional objects which is being viewed by the camera of the smartphone or any other gadget used for the AR application. The three dimensional object that is been viewed on the gadget's screen is adjusted with the location and co-ordinates of the real time image which makes the observer feel that the three dimensional object is a part of the world outside the screen of the gadget. The Vuforia SDK supports the marker system. In the marker system it treats the corners of the two dimensional image as the markers and then uses this markers to place the three dimensional object. This marker system helps to improve the blending of the 3D model with the real world. These markers are often called as "VuMark". Some of the major uses of this SDK are listed as:

- Used for storing the images in Vuforia db. These images will be used as marker for $3 \mathrm{~d}$ object visualization.

- Vuforia database is linked with Unity3d via a license key generated in Vuforia. This key is to be entered into the unity3d to link the database and its images with unity.

- Using Vuforia database to store the $2 \mathrm{~d}$ images of the furniture.

- Create a new project on Vuforia and generate a license key that will be placed in the unity software.

Android SDK: The Android Software Development kit commonly referred as SDK consists of comprehensive development tools. These include a debugger, libraries, a handset emulator based on QEMU, documentation, sample code, and tutorials. Currently supported development platforms include computers running Linux (any modern desktop Linux distribution), Mac OS X 10.5.8 or later, and Windows 7 or later. As of March 2015, the SDK is not available on Android itself, but software development is possible by using specialized Android applications. The Android Software Development Kit (Android SDK) contains the necessary tools to create, compile and package Android applications. Most of these tools are command line based. The primary way to develop Android applications is based on the Java programming language. The Android SDK Platform Tools are a separately downloadable subset of the full SDK, consisting of command-line tools such as adb and fast boot. The Android Debug Bridge (adb) is a tool to run commands on a connected Android device.

Steps to get an android SDK:-

- Obtain the Android SDK.

- Install the Android SDK.

- Open Android SDK Manager.

- Install Android Version and Extras for SDK.

- Obtain Eclipse IDE.

- Run Eclipse for First Time.

- Add ADT Plugin Repository.

- Install ADT Plugin.

Implementation: The marker was image of the sofa that was uploaded in the database of Vuforia which was kept aligned on the wall, when scanned with the application camera gave this 3D model. In the next step, table marker was added along with the sofa marker.

Figure 2: AR projection of the marker sofa

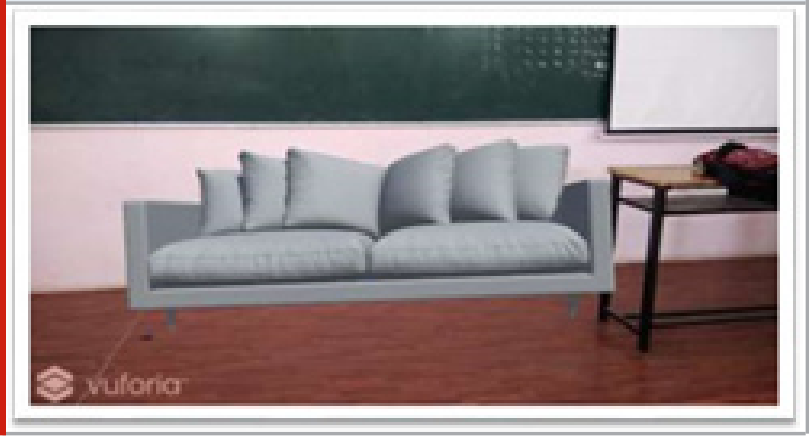

Figure 3: AR projection of table and sofa

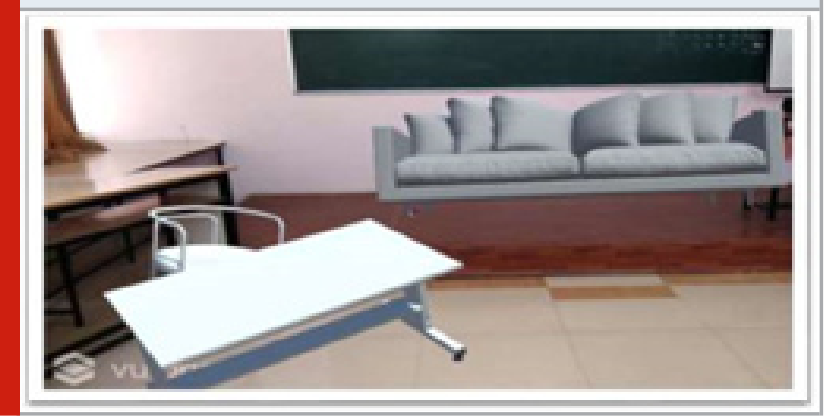

Assumptions of the system

- Initially the application will be made available for limited set of furniture items.

- Each seller will have to provide list of available item set in his shop.

- 3D models for newly arrived furniture items could not be dynamically added.

- The new-users can face difficulty in adjusting the positions of projected $3 \mathrm{~d}$ models

- The user will have to carry a booklet containing images of items.

- The user cannot get the measurements about the furniture item that fits at their place. 
- Mobile camera should be good enough to identify the markers form the image.

- There is a problem with respect to rotation of the 3D object .

- The content is a real problem in Augmented Reality applications; the content is limited and needs to be updated very often.

\section{CONCLUSION AND FUTURE SCOPE}

The application was used to visualize the furniture as a marker and can be used to give the predictions about the real time world coordinates where the object should be placed. It will be possible for the sellers to provide a better service to the customers and grow up their business. The unity $3 \mathrm{~d}$ can be used to know the product and visualize the product in a better and efficient way, thus helping to learn more about the product. It will solve the real life problems faced by the customers at the time of buying the furniture items by allowing the customer to visualize the $3 \mathrm{~d}$ model of the furniture item set which will help customers to make better choice. The application can be used by the seller to advertise their products by playing the promotional video for their shop on scanning the logo of their shop. The application as of now supports android users only. But the Unity3d supports both Android and the iOS so the application can be built for the iOS users also, thus expanding the user base. The application can be expanded to other domains like the educational, medical fields, for other item sets for which it is not possible for customers to carry the items for visualizing.

\section{REFERENCES}

AR interior designer: Automatic furniture arrangement using spatial and functional relationships Jeff K.

T. Tang; WanMan Lau; Kwun-Kit Chan; Kwok-Ho To2014 International Conference on Virtual Systems et Multimedia (VSMM)

A Transitional AR Furniture Arrangement System with
Automatic View Recommendation' Mami Mori, Jason Orlosky, Kiyoshi Kiyokawa, Haruo Takemura Osaka University 2016.

Cathy Hackl, Samantha G. Wolfe. (2017) Marketing New Realities: An Introduction To Virtual Reality ct Augmented Reality Marketing, Branding, \& Communications

Helen Papagiannis (2017). Augmented Human: How technology is shaping the new reality.

Indoor Marker-based Localization Using Coded Seamless Pattern for Interior Decoration Shigeru Saito; Atsushi Hiyama; Tomohiro Tanikawa; Michitaka Hirose 2007 IEEE Virtual Reality Conference

Ishaan Bhola. (2018) Magic: How Augmented Reality Will Change Our World

Jon Peddie. (2018). Augmented Reality: Where We Will All Live

Live Texturing of Augmented Reality Characters from Colored Drawings' Stephane Magnenat, Dat Tien Ngo, Fabio Z 'und, Mattia Ryffel, Gioacchino Noris, Gerhard Rothlin, Alessia Marra, Maurizio Nitti, Pascal Fua, Fellow, IEEE, Markus Gross, Robert W. Sumner 2015

Research on the augmented reality system without identification markers for home exhibition Liyan Chen; XiaoyuanPeng; Junfeng Yao; Hong Qiguan; Chen; Yihan Ma 2016 11th International Conference on Computer Science \& Education (ICCSE)

Sean Morey, John Tinnell. (2016) Augmented Reality: Innovative Perspectives Across Art, Industry, And Academia.

Snap To Reality: (2016) Aligning Augmented Reality to the Real World' Benjamin Nuernberger, Eyal Ofek, Hrvoje Benko, Andy Wilson University of California, USA. 